\title{
INTERNET OF THINGS - IOT A NEW ENVISAGE IN THE DOMAIN OF AUTOMATION
}

\author{
Nidhi Agrawal* \\ School of Computers, IPS Academy, Rau, Indore (M.P.)-452001, India \\ Nidhi.gupta135@gmail.com \\ http://www.ipsacademy.com
}

\begin{abstract}
Internet of things is such a predefined and honorable area which drives us to these present reality situations where each object can play out some undertakings or some tasks while at the same time speaking and communicating with some different items. The world brimming with devices, sensors and different items which will impart and improve human life far and less demanding than at any other time. This paper gives an outline of ebb and flow exploration that takes a shot about IoT regarding engineering, innovation, utilization and applications. It additionally pinpoints every issue connected with the advancements of IoT, after the literature audit of this research work. The fundamental reason for this review is to give all the most recent advancements, their relating patterns and points of interest in the field of IoT in deliberate way. It will be useful for further research.

This literature overviews Internet of Things arranged structures that are sufficiently skilled to enhance the comprehension of related device, innovation, and approach to encourage engineer's prerequisites. Straightforwardly or by implication, the exhibited models propose to tackle genuine issues by building and organization of ground-breaking Internet of Things ideas. Further, examined difficulties have been explored to fuse the lacuna inside the current patterns of structures to persuade the scholastics and ventures get included into looking for the conceivable path outs to able the correct intensity of Internet of Things. A principle commitment of this review paper is that it outlines the current best in class of Internet of Things structures in different areas deliberately.
\end{abstract}

Keywords: Internet of things, framework \& architecture, smart devices, sensors

\section{Introduction}

Prior there was a basic manual method for taking care of machines. Be that as it may, with the progression of innovation, new ways are presented for controlling the machines like computerization and automation. At the bit of a catch, we can get to extensive measure of data because of ability of PCs and the Internet. Everyone needs a moderate and secure approach to control their machines from any brilliant cell phone or Internet association. The Internet of Things (IoT) is the system of physical articles or "things" implanted with hardware, programming, sensors and network to empower it to accomplish more noteworthy esteem and administration by trading information with the maker, administrator or other associated gadgets. Everything is interestingly recognizable through its installed computing framework however can interoperate inside the current Internet foundation. Internet of Things is the following enormous insurgency of the world on digitalization of commercializing different modules/items. Everything is related with the web, some includes controlling and some includes checking the parameters from anyplace. The Internet of Things is today's most drifting innovation that remains close by wearables and mechanical technology. The Internet of Things (IoT) assumes an exceptional job in all parts of our day by day lives. It covers numerous fields including healthcare, automobiles, amusements, mechanical machines, sports, homes, etc. The inescapability of IoT facilitates some regular exercises, improves the manner in which individuals cooperate with nature and environment, and expands our social connections with other individuals and items.

This all-encompassing vision, be that as it may, raises likewise a few concerns, similar to which dimension of security the IoT could give? what's more, how it offers and secures the protection of its clients? Creating applications for the IoT could be a testing assignment because of a few reasons; (i) the high multifaceted nature of disseminated figuring, (ii) the absence of general rules or systems that handle low dimension correspondence and improve abnormal state usage, (iii) numerous programming dialects, and (iv) different correspondence conventions. It includes engineers to deal with the foundation and handle both programming and equipment layers along with saving all utilitarian and non-useful programming prerequisites.

This multifaceted nature has prompted a brisk development as far as presenting IoT programming systems that handle the previously mentioned difficulties. Internet of Things is one where consistently gadget turned out to be more intelligent, consistently handling winds up savvy, and consistently correspondence ends up instructive. While the Internet of Things is as yet looking for its own shape, its belongings have just gazed in making mind 
boggling strides as a widespread arrangement media for the associated situation. Design's explicit investigation does dependably clear the compliance of related field. The absence of generally speaking design learning is by and by opposing the scientists to get past the extent of Internet of Things driven methodologies. This writing overviews Internet of Things situated designs that are sufficiently competent to enhance the comprehension of related instrument, innovation, and system to encourage designer's necessities.

Straightforwardly or in a roundabout way, the displayed structures propose to take care of genuine issues by building and sending of incredible Internet of Things ideas. Further, examine difficulties have been explored to consolidate the lacuna inside the current patterns of designs to persuade the scholastics furthermore, ventures get included into looking for the conceivable route outs to adept the correct power Internet of Things. A principle commitment of this study paper is that it condenses the current best in class of Internet of Things models in different areas efficiently.

Internet of Things (IOT) has given a chance to fabricate incredible mechanical framework and applications by utilizing the developing omnipresence of RFID, remote, portable and sensor gadgets. Numerous modern IOT applications have been progressively created and sent as of late. Presently controlling and checking assumes a primary job in our everyday life. We can screen and control everything utilizing trend setting innovations. Remote access is a superb element that came on account of fast web. The fundamental target of proposed framework is to give an innovation arranged and minimal effort framework to make a propelled industry for the individuals who far from their industry and need to control gadgets. Internet of Things is where consistently gadgets turned out to be more intelligent, consistently preparing winds up insightful, and consistently correspondence ends up educational.

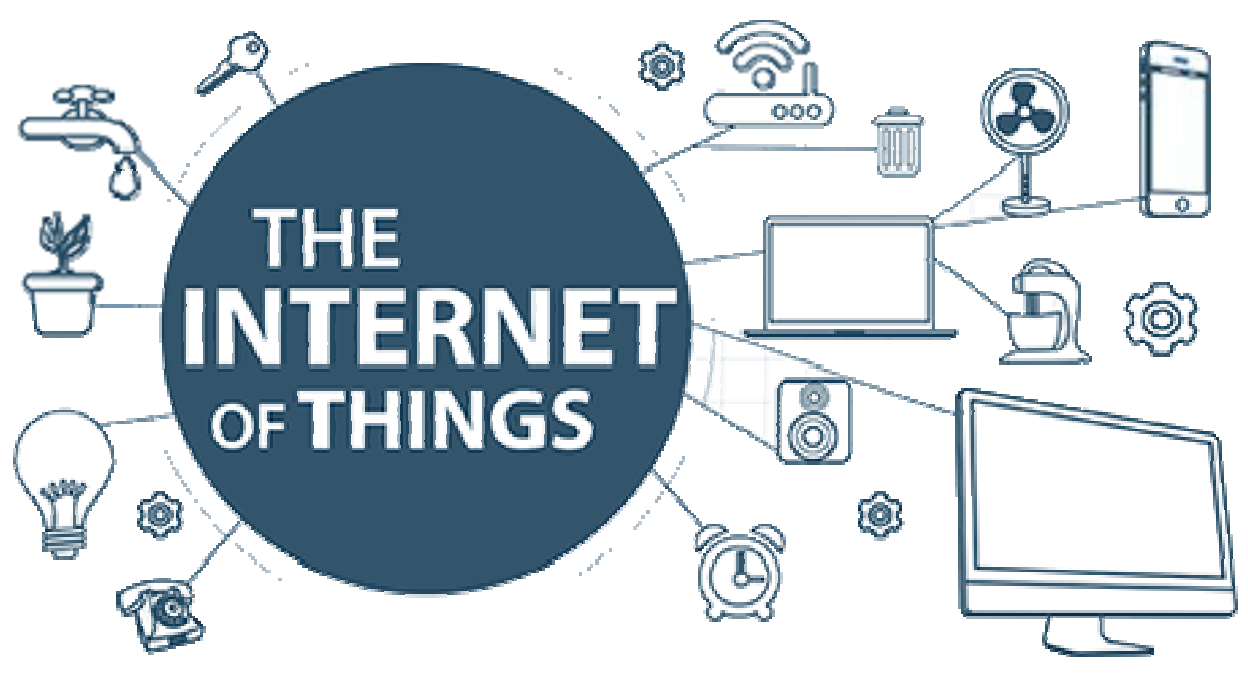

Figure 1: Framework of Internet of Things

\section{Literature survey}

Internet of Things (IoT) refers to the stringent connectedness between digital and physical world (Atzori et al., 2010; Sterling, 2005; Internet Reports, 2005). Various researchers have described IoT in multitude forms:

* "a dynamic global network infrastructure with self configuring capabilities based on standard and interoperable communication protocols where physical and virtual 'Things' have identities, physical attributes, and virtual personalities and use intelligent interfaces, and are seamlessly integrated into the information network" (Kranenburg, 2008).

* "3A concept: anytime, anywhere and any media, resulting into sustained ratio between radio and man around 1:1” (Srivastava, 2006).

* "Things having identities and virtual personalities operating in smart spaces using intelligent interfaces to connect and communicate within social, environmental, and user contexts" (Networked Enterprise \& RFID \& Micro \& Nanosystems, 2008). The semantic meaning of "Internet of Things" is presented as "a worldwide network of interconnected objects uniquely addressable, based on standard communication protocols". We will consider the definition provided by the ITU:

* "A global infrastructure for the information society enabling advanced services by interconnecting (physical and virtual) things based on, existing and evolving, interoperable information and communication technologies” (ITU work on Internet of things, 2015). 
According to Gartner, 25 billion gadgets will be associated with the web by 2020 and those associations will encourage the utilized information to break down, preplan, oversee, and settle on shrewd choices self-rulingly. The US National Intelligence Council (NIC) has set out IoT as one of the six "Disruptive Civil Advances" (National Intelligence Council, 2008). In this setting, we can see that benefit a few segments, for example, transportation, savvy city, brilliant domotics, keen wellbeing, governance, helped living, e-training, retail, coordination's, agribusiness, computerization, mechanical assembling, and business/ process the executives and so on., are as of now getting profited from different design types of IoT (Gubbia et al., 2013; Miorandi et al., 2012; Giusto et al., 2010).

IoT engineering might be treated as a framework which can be physical, virtual, or a crossover of the two, comprising of a gathering of various dynamic physical things, sensors, actuators, cloud administrations, explicit IoT conventions, correspondence layers, clients, designers, and venture layer. Specific models do go about as a rotate part of IoT explicit framework while encouraging the deliberate methodology toward disparate segments coming about answers for related issues. An all-around characterized type of IoT engineering is right now accessible for information reason:

* "a dynamic worldwide system foundation with self-designing capacities dependent on standard and interoperable correspondence conventions where physical and virtual 'Things' have characters, physical qualities, and virtual identities and utilize canny interfaces, and are consistently incorporated into the data arrange" (Kranenburg, 2008).

Li Da Xu, Wu He, and Shancang Li [1]: This paper surveys the ebb and flow research of IoT, key empowering innovations, major IoT applications in ventures, and recognizes look into patterns and difficulties. A fundamental commitment of this audit paper is that it abridges the current cutting edge IoT in enterprises efficiently.

AlaaAlhamoud, Felix Ruettiger, Andreas Reinhardt, Frank Englert, Daniel Burgstahler, Doreen Bohnstedt, Christian Gottron and Ralf Steinmetz [2]: This paper presents structure for acknowledging vitality productive brilliant homes dependent on remote sensor systems and human movement recognition. Their work depends on the possibility that the greater part of the client exercises at home are identified with an arrangement of electrical machines which are important to play out these exercises. Hence, they demonstrate how it is conceivable to identify the client's present movement by observing his fine-grained machine level vitality utilization. This connection among exercises and electrical machines makes it conceivable to recognize apparatuses which could be squandering vitality at home. Our structure is sorted out in two segments. On one hand, the movement discovery structure which is in charge of recognizing the client's present action dependent on his vitality utilization.

Kemal Akkaya, Ismail Guvenc, Ramazan Aygun, Nezih Pala and Abdullah Kadri [3]: This paper studies the current and takes a shot at inhabitancies checking and multi-modular information combination procedures for keen business structures. The objective is to set out a system for future research to misuse the spatiotemporal information got from at least one of different IoT gadgets, for example, temperature sensors, reconnaissance cameras, and RFID labels that might be as of now being used in the structures.

Andrea Zanella, Nicola Bui, Angelo Castellani, LorenzoVangelista, and Michele Zorzi [4]: This paper concentrates explicitly to an urban IoT framework that, while as yet being a significant general class, are portrayed by their particular application area. Urban IoTs, indeed, are intended to help the Smart City vision. This goes for abusing the most exceptional correspondence innovations to help included esteem administrations for the organization of the city and for the residents. This paper henceforth gives an exhaustive review of the empowering advancements, conventions, and engineering for an urban IoT.

Ala Al-Fuqaha, Mohsen Guizani, Mehdi Mohammadi, Mohammed Aledhari, and Moussa Ayyash [5]: This paper gives a review of the Internet of Things (IoT) with accentuation on empowering advancements, conventions, and application issues. The IoT is empowered by the most recent improvements in RFID, brilliant sensors, correspondence advancements, and Internet conventions. The fundamental introduce is to have keen sensors work together specifically without human inclusion to convey another class of utilizations. The present unrest in Internet, versatile, and machine-to-machine (M2M) innovations can be viewed as the primary period of the IoT. In the coming years, the IoT is relied upon to connect different innovations to empower new applications by associating physical protests together in help of smart basic leadership. This paper gives a level diagram of the IoT. At that point give a review of some specialized subtle elements that relate to the IoT empowering advances, conventions, and applications. Contrasted with other overview papers in the field, our goal is to give a more intensive outline of the most significant conventions and application issues. 


\section{Applications of IOT}

Smart agriculture: - We can transform the world though iot. In agriculture iot play major role. IoT-based smart farming, a system is built for monitoring the crop field with the help of sensors (light, humidity, temperature, soil moisture, etc.) and automating the irrigation system. The farmers can monitor the field conditions from anywhere. IoT-based smart farming is highly efficient when compared with the conventional approach.

Smart healthcare: - we can decrease many diseases with the help of iot devices. If we monitor patient health on daily basis so we can handle many diseases. If we use iot devices we can provide health services for emergency cases very quickly. The rise of IoT in healthcare has potentially lifesaving applications by collecting data from bedside devices and viewing patient information and diagnosing in real-time. IoT in healthcare improves the patient's experience and provider's ROI.

Security Home, Security \& surveillance: -In current situation everyone need security from unauthorised access, theft and fraud. Right security protects company data and details. Iot is a right solution for preventing data from unauthorised access.

Intelligent transportation: Today transportation in our country improving very gradually. Transport system made not only use of concrete and steel but also use of technology. We are facing many problems in transportation. We can control transportation problem with the help of iot. With the help of intelligent transportation system, we provide innovative services relating to different modes of transport and enable various users to be better informed and make safer, more coordinated and smart use of transport.

Smart energy management: Energy is very important facet for any household, industries, agriculture and so on. Very important for us to managing the energy efficiently. conserving it intelligently for appliances is very much important. The energy usage is directly affected with Coal, oil and so towards power generation.

Smart environment: smart environment and healthy atmosphere increase productivity of every industries. Today pollution is very big problem facing in all over the world. We can reduce this problem with the help of iot. We can measure the level of pollution with the help of smart sensors.

Smart governance: smart governance plays major role for our country. Today Andhra Pradesh government implementing iot in civil supplies. With the help of smart governance, we increase transparency, speediness and productivity.

WiFi products: Today Wi-Fi is a popular short-range radio technology widely used. We use muddle up of technology will be used for linking all devices simultaneously. Wi-Fi is one candidate technology for capillary radio - connecting tiny devices into the Internet of Things - but some adaptations will be needed.

RFID manufacturing and solutions: RFID technology is essential for business applications is the concept of the so called "internet of things". We add RFID tag for goods, pallets and finished items can speed manufacturing, logistics and service operations. wide range of Many business use application tracking assets activities more efficient. RFID tags can be used to tell applications what things are, where things are, if things have moved, who moved them or used them. 
Table 1: Security Features

\begin{tabular}{|c|c|}
\hline Security feature & Implementation in embedded devices \\
\hline Secure boot & $\begin{array}{l}\text { Achieved using cryptographically signed code from the manufacturer along with } \\
\text { hardware support to verify code is authenticated. This ensures that the firmware } \\
\text { has not been altered. }\end{array}$ \\
\hline $\begin{array}{l}\text { Secure code } \\
\text { updates }\end{array}$ & $\begin{array}{l}\text { A method of secure code updates that ensure that the code on the device can be } \\
\text { updated for bug fixes, security patches, etc. Use of signed code (secure boot) } \\
\text { ensures that malicious code cannot be introduced into the system. }\end{array}$ \\
\hline Data Security & $\begin{array}{l}\text { Prevent unauthorized access to the device, encrypted data storage and/or } \\
\text { encrypted communication. }\end{array}$ \\
\hline Authentication & $\begin{array}{l}\text { All communication with the device should be authenticated using strong } \\
\text { passwords (at a minimum) or use of an authentication protocol such as X.509 or } \\
\text { Kerberos. }\end{array}$ \\
\hline $\begin{array}{l}\text { Secure } \\
\text { communication }\end{array}$ & $\begin{array}{l}\text { Communication to/from the device needs to be secured using encrypted } \\
\text { communication (SSH, SSL, etc.). Care must be taken to avoid the use of insecure } \\
\text { encryption algorithms. I.e., } 40 \text { bit encryption keys that were once state-of-the- } \\
\text { art and still used in many embedded devices are no longer considered secure. }\end{array}$ \\
\hline $\begin{array}{l}\text { Protection against } \\
\text { cyber attacks }\end{array}$ & $\begin{array}{l}\text { Embedded firewalls provide a critical layer of protection against attacks. A } \\
\text { firewall can limit communication to only known, trusted hosts, blocking hackers } \\
\text { before they can even launch an attack. It is essential to provide a layer of } \\
\text { defense to protect against common attacks such as packet flood attacks, buffer } \\
\text { overflow attacks and known protocol exploits. A firewall can implement many of } \\
\text { these protections, but some must be built into the embedded applications. }\end{array}$ \\
\hline $\begin{array}{l}\text { Intrusion detection } \\
\& \text { security } \\
\text { monitoring }\end{array}$ & $\begin{array}{l}\text { Existing embedded devices can be attacked and no one would ever know. A } \\
\text { hacker could execute thousands or millions of invalid login attempts without the } \\
\text { attack being reported. Embedded devices must be able to detect and report } \\
\text { invalid login attempts and other potentially malicious activities. NOTE: } \\
\text { Monitoring requirements for embedded devices are very different from } \\
\text { enterprise requirements. The IDS requirements for embedded devices will } \\
\text { depend upon the protocols supported by the device. }\end{array}$ \\
\hline $\begin{array}{l}\text { Embedded security } \\
\text { management }\end{array}$ & $\begin{array}{l}\text { Integration with a security management system allows security policies to be } \\
\text { updated to mitigate against known threats. }\end{array}$ \\
\hline $\begin{array}{l}\text { Device tampering } \\
\text { detection }\end{array}$ & $\begin{array}{l}\text { Some new processor/board designs include device tamper detection capabilities. } \\
\text { They provide the ability to detect that the seal on the device enclosure has been } \\
\text { broken, indicate that someone may be attempting to tamper with the device. }\end{array}$ \\
\hline
\end{tabular}

Convergence platform: The collaboration of IoT and cloud technologies will hopefully increase our quality of life globally through digital sophistication and is widely applicable to a range of industries, for example, healthcare systems, atmosphere monitoring, agriculture, home/office automation, transportation, infrastructure, industry, social networks, entertainment, and security systems. Cloud computing and Internet of Things (IoT) are two very different technologies that are both already part of our life. Their adoption and use are expected to be more and more pervasive, making them important components of the Future Internet. 


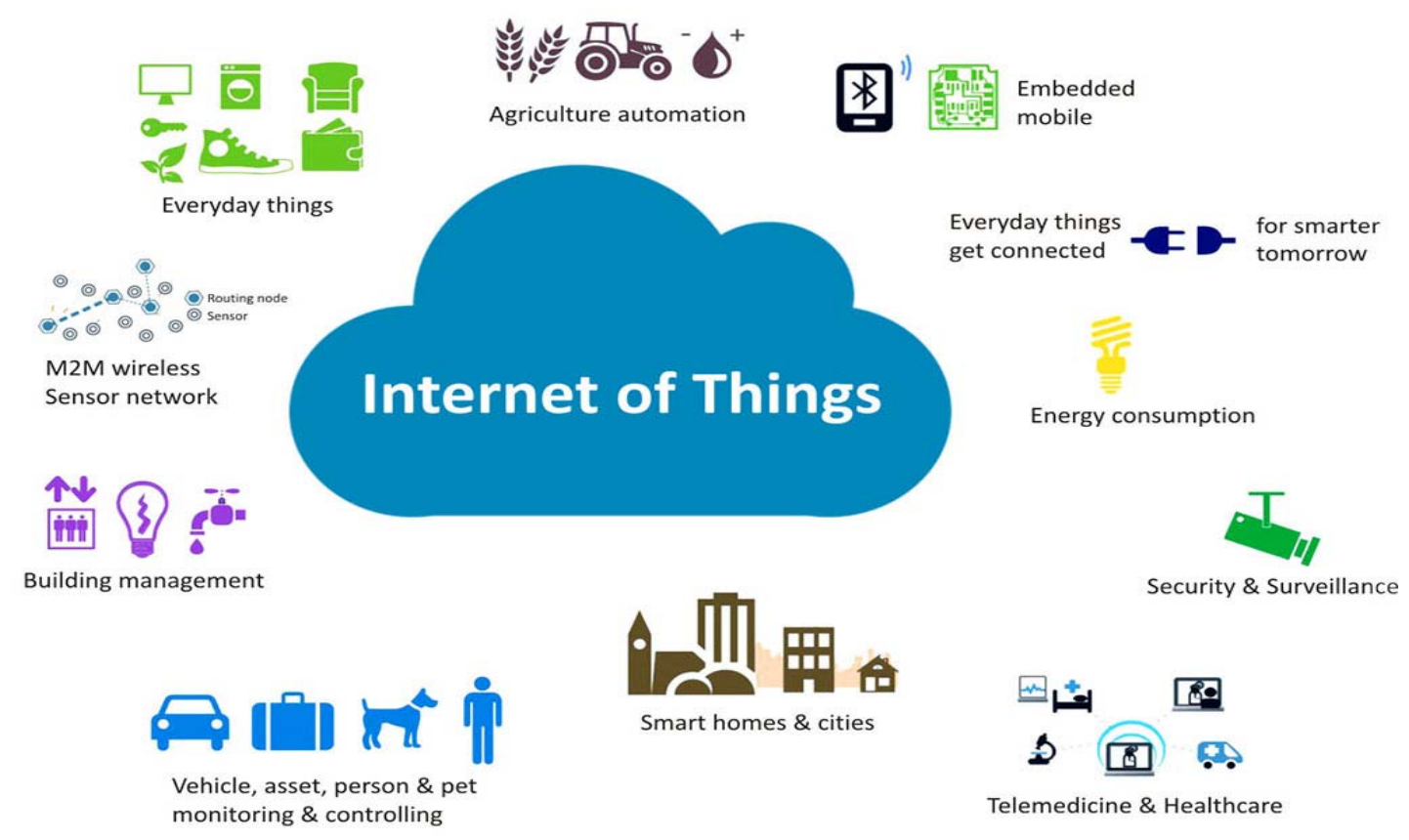

Figure 1: Application of Internet of Things

\section{Conclusion}

The IoT showcase is developing quickly and as a result the consideration has moved from proposing single IoT components and conventions towards application stages with the end goal to distinguish structures supporting the standard IoT suites of controls and conventions. This scrutinization has secured a subset of economically accessible structures and stages for creating modern and consumer based IoT applications. The chose structures have a similar plan rationality as far as distinguishing cloud-based applications by bringing together circulated information sources. In any case, they pursued different methodologies with the end goal to apply this logic. A relative investigation of the structures was led dependent on the engineering, equipment similarity, programming prerequisites, and security. We featured on the safety efforts of every structure as checking the different security highlights and resistance against assaults is a standout amongst the most vital contemporary issues confronting the Internet of Things.

Internet of Things relies upon Internet, sensors innovation which makes the correspondence conceivable among gadgets by actualizing diverse conventions. In the wake of doing the literary study some significant issues are watched, similar to the intruded-on availability among gadgets affecting the correspondence. Additionally, there is similarity issue in gadgets. Security of gadgets amid correspondence process and security of correspondence channel or connection is additionally a noteworthy issue. Heaps of work is to be improved the situation the advancement and advancement of this field; still there is more work to do, more institutionalization of innovation, conventions and equipment are required to make totally solid and secure area of Internet of Thing. Some worldwide rules ought to be utilized for this reason. What's to come is absolutely relies upon Internet of Thing, so parcel of activity at execution level. With the end goal to determine security issues in IoT space we propose to execute the idea of Block Chain in IoT. We will have profound exchange on principals and execution of Block Chain in our further works.

The Internet has demonstrated its reality in our lives, from collaborations at a virtual dimension to social connections. The IoT has included another potential into web by empowering correspondences among items and human, making a more astute and smarter planet. This has driven the vision of "whenever, anyplace, anyway, anything" correspondences for all intents and purposes in obvious sense. 


\section{References}

[1] Li Da Xu, Wu He, and Shancang Li,” Internet of Things in Industries: A Survey” IEEE transactions on industrial informatics, vol. 10, no. 4, November 2014

[2] Alaa Alhamoud, Felix Ruettiger, Andreas Reinhardt, Frank Englert, Daniel Burgstahler, Doreen Bohnstedt, Christian Gottron and Ralf Steinmetz," SMARTENERGY.KOM: An Intelligent System for Energy Saving in Smart Home”, 3rd IEEE international workshop on global trends 2014.

[3] Kemal Akkaya, Ismail Guvenc, Ramazan Aygun, Nezih Pala, Abdullah Kadri,” IOT-based Occupancy Monitoring Techniques for Energy-Efficient Smart Buildings”,2015 IEEE wireless communication and networking conference.

[4] Andrea Zanella, Nicola Bui, Angelo Castellani,Lorenzo Vangelista, and Michele Zorzi, "Internet of Things for Smart Cities” IEEE Internet of things journal, Vol. 1, No. 1, FEBRUARY2014.

[5] Ala Al-Fuqaha, Mohsen Guizani, Mehdi Mohammadi, Mohammed Aledhari, and Moussa Ayyash,” Internet of Things: A Survey on Enabling Technologies, Protocols, and Applications”, IEEE communication surveys \& tutorials, vol. 17, no. 4, fourth quarter 2015.

[6] Akyildiz, I.F., Su, W., Sankarasubramaniam, Y., Cayirci, E., 2002. Wireless sensor networks: a survey. Comput. Netw. 38 (4), $393-$ 422.

[7] Arsénio, A., Serra, H., Francisco, R., Nabais, F., Andrade, J., Serrano, E., 2014. Internet of intelligent things: bringing artificial intelligence into things and communication networks. Stud. Comput. Intell. 495, 1-37. 